\title{
Pharmacological activation of rev-erba suppresses LPS-induced macrophage M1 polarization and prevents pregnancy loss
}

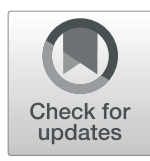

Liyuan Cui ${ }^{1,2 \dagger}$, Feng $\mathrm{Xu}^{1,2+}$, Songcun Wang ${ }^{1,2}$, Xinyi Li ${ }^{1,2}$, Haiyan $\operatorname{Lin}^{3}$, Yan Ding ${ }^{1,2,4^{*}}$ and Meirong Du ${ }^{1,2,5,6^{*}}$

\begin{abstract}
Background: Circadian rhythm is an important player for reproduction. Rev-erba, a significant clock gene, is involved in regulating cell differentiation, inflammation and metabolism. Macrophage polarization plays crucial roles in immune tolerance at the maternal-fetus interface, which also modulates the initiation and resolution of inflammation. Alteration of macrophage polarization induces adverse pregnancy outcomes such as infertility, recurrent spontaneous abortion and preterm labor.

Results: Decidual macrophages from LPS-induced mice abortion model displayed M1-like bias, accompanied by decreased expression of Rev-erba. SR9009, an agonist of Rev-erba, may reduce lipopolysaccharide (LPS)-induced M1 polarization of macrophages via activation of PI3K but not NF-KB signaling pathway. Furthermore, SR9009 could reduce M1-like polarization of decidual macrophages induced by LPS and attenuate LPS-induced resorption rates in mice model.

Conclusions: Both in vivo and in vitro experiments demonstrated that the pharmacological activation of Rev-erba using SR9009 could attenuate the effect of LPS on macrophage polarization and protect pregnancy. This study may provide a potential therapeutic strategy for miscarriage induced by inflammation.
\end{abstract}

Keywords: Rev-erba, Decidual macrophages, M1/M2 polarization, Pregnancy

\section{Background}

Circadian rhythm is an endogenous free running cycle lasting near $24 \mathrm{~h}$. In mammals, suprachiasmatic nuclei $(\mathrm{SCN})$ is a master pacemaker coordinating the environmental changes to physiological activities [1]. Meanwhile, SCN synchronizes activities of other nucleus and peripheral organs by directly synaptic transmission or secreting peptides [2]. In molecule level, the circadian rhythm is regulated by clock genes in a transcriptionaltranslational loop. Brain and muscle ARNT-like 1

\footnotetext{
* Correspondence: ding_yan@fudan.edu.cn; dmrlq1973@sina.cn

†Liyuan Cui and Feng Xu contributed equally to this work.

${ }^{1} \mathrm{NHC}$ Key Lab of Reproduction Regulation (Shanghai Institute of Planned

Parenthood Research), Hospital of Obstetrics and Gynecology, Fudan University Shanghai Medical College, Shanghai 200090, China

Full list of author information is available at the end of the article
}

(Bmal1) and circadian locomotor output cycles kaput (Clock) are two core clock genes. BMAL1-CLOCK heterodimers modulate the transcription of clock genes with E-box sequence such as Per1-3, Cry1-2, Rev-erbo. In the transcriptional-translational feedback loop, Reverb $\alpha$ is an important clock gene and its protein directly represses the transcription of Bmal1 [1,3]. In addition, Rev-erb $\alpha$, as a transcription factor, is reported to be involved in regulation of behavior rhythm, metabolism, autophagy and inflammation [4-7]. Therefore, Rev-erb $\alpha$ may be an important therapeutic target of multiple diseases.

Circadian rhythm plays crucial roles in reproduction. Shift work is a common form of circadian rhythm disruption. Epidemiological studies have demonstrated that shift work increased the risk of infertility, menstrual

(c) The Author(s). 2021 Open Access This article is licensed under a Creative Commons Attribution 4.0 International License, which permits use, sharing, adaptation, distribution and reproduction in any medium or format, as long as you give appropriate credit to the original author(s) and the source, provide a link to the Creative Commons licence, and indicate if changes were made. The images or other third party material in this article are included in the article's Creative Commons licence, unless indicated otherwise in a credit line to the material. If material is not included in the article's Creative Commons licence and your intended use is not permitted by statutory regulation or exceeds the permitted use, you will need to obtain permission directly from the copyright holder. To view a copy of this licence, visit http://creativecommons.org/licenses/by/4.0/ The Creative Commons Public Domain Dedication waiver (http://creativecommons.org/publicdomain/zero/1.0/) applies to the data made available in this article, unless otherwise stated in a credit line to the data. 
dysregulation and miscarriage $[8,9]$. It has reported that knockout of clock genes such as Bmal1, clock, per1 and Rev-erb $\alpha$ in mice showed a series of adverse pregnancy outcomes like implantation failure and miscarriage [3, 10, 11]. Thus, disruption of circadian rhythm may be an important cause of adverse pregnancy outcomes. As is well known that intrauterine inflammation can destroy immunologic microenvironment and trigger spontaneous abortion and preterm birth [12]. Previous studies proved pharmacological activation of Rev-erbo suppressed the inflammatory response [7]. Whether Rev-erb $\alpha$ is involved in maintaining the balance of immunologic microenvironment by inflammatory regulation remains unclear.

During normal pregnancy, maternal immune cells take important parts in immune tolerance to semi-allogeneic fetus. Decidual macrophages $(\mathrm{dM} \phi \mathrm{s})$ are the second abundant immune cells next to natural killer (NK) cells in the decidua, and play roles in maintaining the balance of immunologic microenvironment at maternal-fetal interface [13]. $\mathrm{DM} \phi s$ are characterized by high plasticity, whose function can be altered on the basis of the different tissue microenvironment. Parallel to Th1/Th2 paradigm, macrophages are originally divided into classically activated (M1) and alternatively (M2) populations according to their function and production of cytokines $[13,14]$. M1 population is characterized by proinflammatory phenotype and generated in the exposure of pathogen such as LPS and pro-inflammatory cytokines like interferon- $\gamma$ (IFN- $\gamma$ ) and tumor necrosis factor (TNF)- $\alpha$. M1 population shows high expression of CD80, CD86, iNOS and is more effective at microbicidal properties and switching $\mathrm{T}$-cell responses to Th1 immune response. M2 population exhibits antiinflammation phenotype and is induced in the presence of interleukin (IL)-4, IL-13, or IL-10. M2 population is characterized by high expression of CD163, CD206, CD209 and Arg1, and plays roles in immunosuppression, tissue remodeling and promotion of immunomodulatory profile. Once the balance of M1/M2 populations is disrupted, the critical events of pregnancy like decidualization and vascular remodeling display pathological behaviors $[13,15]$. Thus, the polarization of macrophage is significant for successful pregnancy. Circadian rhythm also exists in macrophages. The expression of cytokines in macrophages shows fluctuation of circadian rhythm and Rev-erb $\alpha$ modulates the expression of some cytokines in macrophages [16-18]. However, whether Reverb $\alpha$ modulates polarization of macrophage is undefined.

LPS is the primary constituent of the outer membrane of Gram-negative bacteria and has been regarded as an immune stimulatory molecule. It can elicit proinflammatory response in many cells such as macrophage and neutrophil [19]. Thus, it has been used to construct many inflammatory disease models like miscarriage and endometritis. Toll-like receptor (TLR) 4, a member of TLR family, recognizes the microbeassociated molecular patterns, including LPS [20]. The activation of TLR4 triggers the activation of NF- $\kappa B$ signaling pathway by recruiting downstream adaptors and then induces the expression of inflammatory cytokines. Previous studies proved LPS suppressed the expression of clock genes including Rev-erb $\alpha$ [7, 21]. Moreover, pharmacological activation of Rev-erb $\alpha$ could suppress LPS-induced inflammatory response [21]. Therefore, there may be an interaction between inflammation induced by LPS and Rev-erb $\alpha$. But whether Rev-erb $\alpha$ alters the changes of macrophages induced by LPS and attenuates the effect of inflammation on pregnancy remain unclear.

In this study, we observed the phenotype of $\mathrm{d} M \phi s$ and the expression of Rev-erb $\alpha$ in $\mathrm{dM} \phi$ s from mice in LPSinduced abortion model. Then, we analyzed the phenotype changes of differentiated macrophages from U937 under the stimulation of LPS and the effect of SR9009, an agonist of Rev-erbo, on phenotype changes of differentiated macrophages from U937 treated with LPS. Moreover, the protective role of SR9009 on abortion induced by LPS was explored. Our study may provide some novel strategies for miscarriage induced by inflammation.

\section{Results}

M1-like polarization and downregulated expression of rev-erba in $\mathrm{dM} \varphi \mathrm{s}$ were observed in LPS-induced mice abortion model

LPS has been widely used to establish various animal models, such as inflammatory diseases and spontaneous abortion [22, 23]. To investigate the effect of LPS on polarization of $\mathrm{dM} \phi \mathrm{s}$ in mice, pregnant mice were injected with LPS on E7.5. As shown in Fig. 1A-B, the expression of M1 makers (CD11c, CD86 and iNOS) was increased, whereas the expression of M2 markers (Arg1) was decreased in $\mathrm{F} 4 / 80^{+} \mathrm{dM} \phi$ s from LPS-induced mice abortion model compared with those from control mice. LPS promoted the secretion of pro-inflammatory cytokines in macrophages [24]. We also detected the mRNA level of pro-inflammatory cytokines (IL- $1 \beta$, IL- 6 , TNF- $\alpha$, IFN- $\gamma$, IL-17a) was significantly upregulated in decidual tissue from LPS-treated mice than those in control mice (Fig.1C) Additionally, the mRNA level of Th2 cytokines (IL-5, TGF $\beta$ ) was significantly downregulated in decidual tissue from LPS-treated mice than those in control mice (Fig.1C). Consistent with our previous studies that LPS treatment in vitro suppress the expression of Rev-erb $\alpha$ in human endometrial stroma cells (ESCs) [21], the expression of Rev-erbo in decidual tissue from mice treated with LPS was dramatically downregulated 


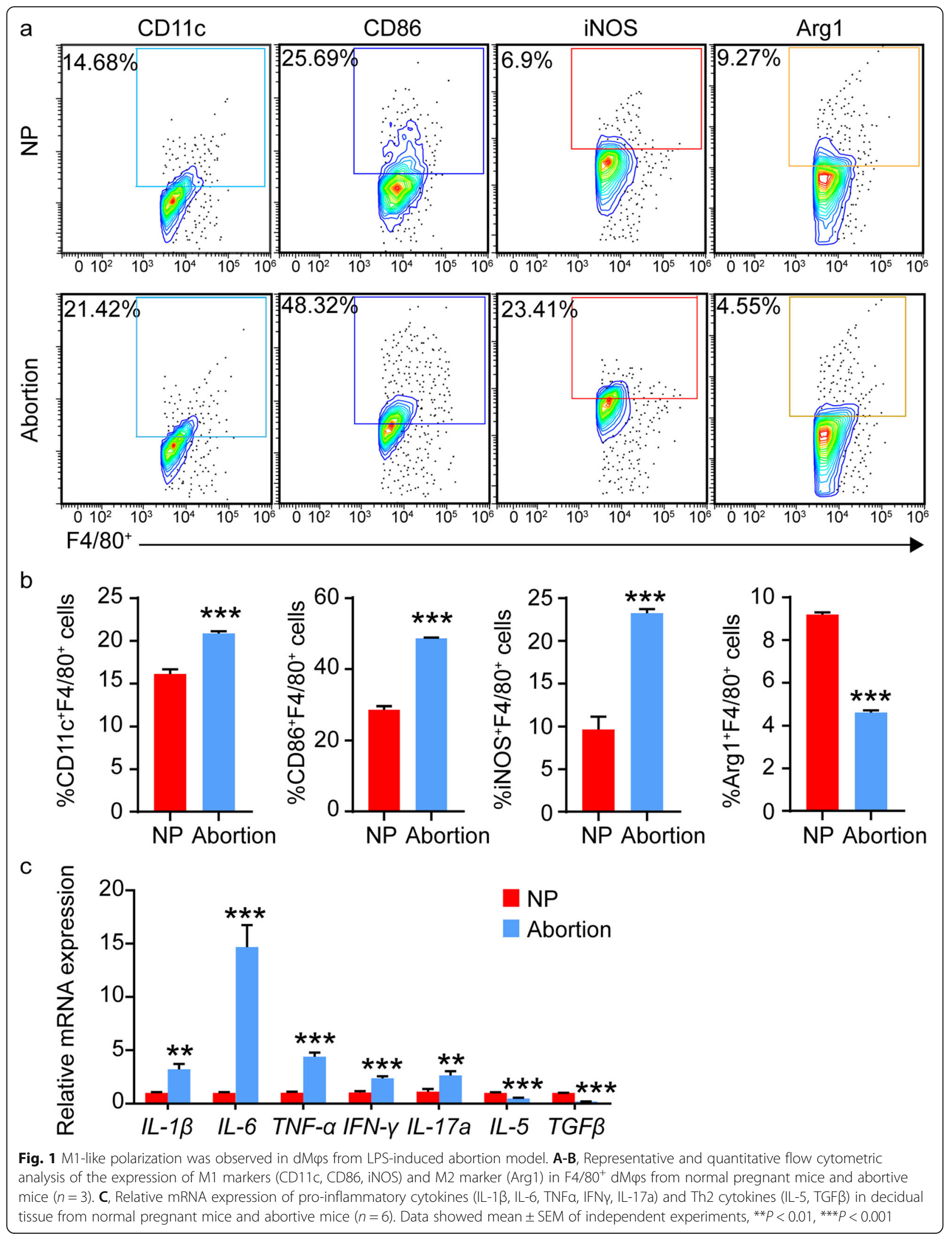


(Fig.2A). The immunofluorescence staining further proved that LPS administration significantly decreased the expression of Rev-erb $\alpha$ in $\mathrm{F} 4 / 80^{+} \mathrm{dM} \phi \mathrm{s}$ of pregnant mice (Fig.2B-C).

\section{Activation of rev-erba reduced the effect of LPS on macrophage polarization}

Excessive inflammation due to bacterial infection is a common cause of early pregnancy loss [25]. LPS, a component of Gram-negative bacteria, induced M1-like polarization of $\mathrm{dM} \phi \mathrm{s}$ and decreased the expression of Rev-erb $\alpha$ in $\mathrm{dM} \phi$ s from mice abortion model. We wondered if LPS treatment could also affect the expression of Rev-erbo and phenotype changes in differentiated macrophages from U937. As shown in Fig. 3A-E, LPS administration significantly decreased the expression of Rev-erb $\alpha$ in differentiated macrophages from U937 with M1 dominance. We then investigate if activation of Reverb $\alpha$ could reduce LPS-induced M1 polarization of differentiated macrophages from U937. The results in Fig. 3B-E demonstrated that administration of SR9009 could reduce the expression of M1 marker (CD86) and increase the expression of M2 markers (CD163, CD206 and CD209), suggesting that activation of Rev-erb $\alpha$ alleviated LPS-induced M1 polarization of differentiated macrophages from U937. We then further explore the potential downstream signaling pathway involved in the regulation. Previous studies proved PI3K/Akt signaling pathway participated in macrophage polarization [26, 27]. To prove Rev-erb $\alpha$ might regulate phenotype of macrophages via PI3K signaling pathway, we used LY294002, a PI3K inhibitor, to block PI3K signaling pathway and detected the phenotype changes of differentiated macrophages from U937. We found that inhibition of PI3K by LY294002 suppressed the effect of SR9009 on LPS-induced M1 polarization, suggesting that the PI3K signaling pathway may be involved in the regulation of Rev-erb $\alpha$ on LPS-induced M1 polarization (Fig.3B-E). We have previously reported that LPS increased the expression of TLR4 and then recruited downstream molecules to activate the NF- $\mathrm{kB}$ signaling pathway and SR9009 suppressed the activation of NF- $\kappa B$ induced by LPS in uterine endometrial stromal cells [21]. Unexpected, SR9009 could not repress the increased expression of TLR4 and NF- $\mathrm{B}$ induced by LPS in differentiated macrophages from U937 (Fig.3F). In addition, SR9009 inhibited LPS-induced ROS production in differentiated macrophages from U937, which could also be reversed by the PI3K inhibitor (Fig.3G). Thus, SR9009 may attenuate the M1 polarization induced by LPS treatment via the PI3K signaling pathway but not the NF- $\mathrm{kB}$ signaling pathway.
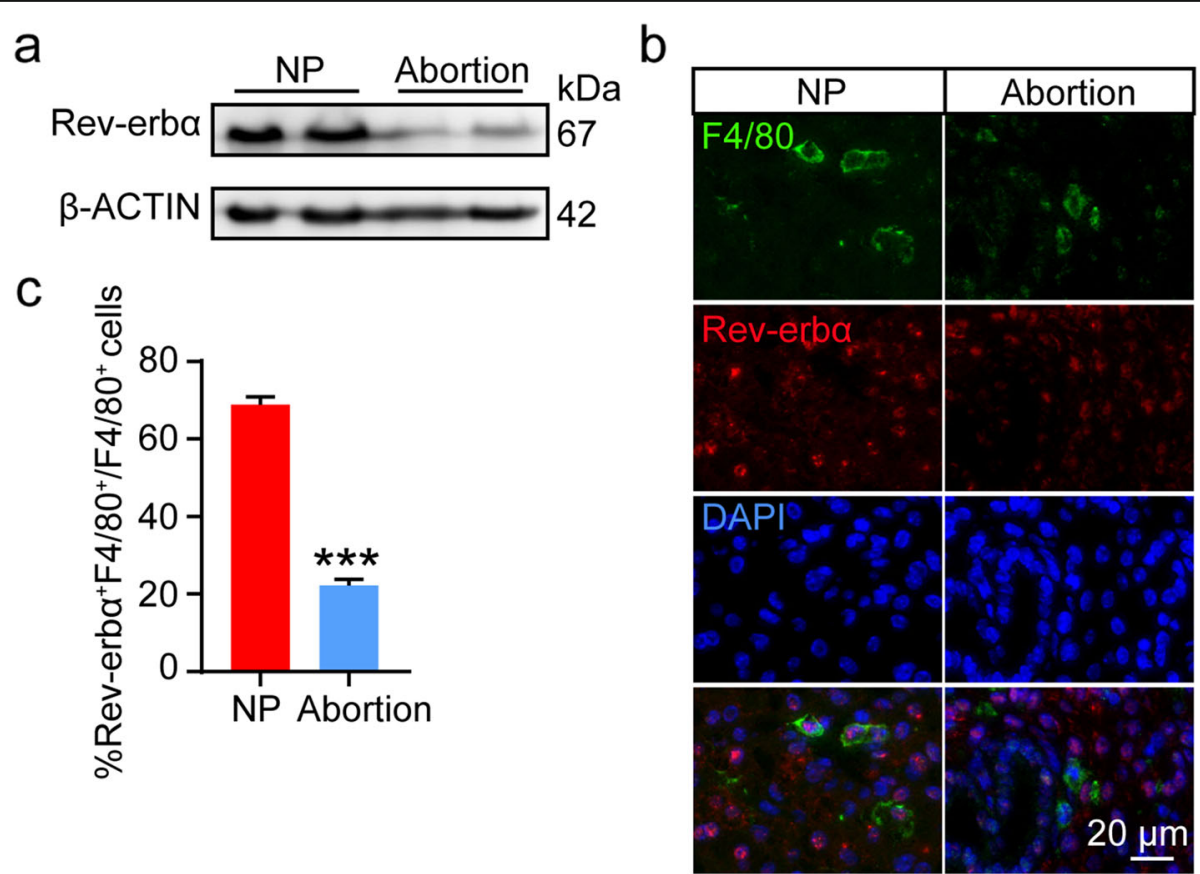

Fig. 2 Downregulated expression of Rev-erba was observed in dM $\varphi$ s from LPS-induced abortion model. A, Protein level of Rev-erba in decidual tissue from normal pregnant mice and abortive mice was analyzed by western blot. The displayed blots were cropped blots. Full-length blots were presented in Supplementary Fig. 1. B, Expression of Rev-erba in F4/80 $\mathrm{dM} \varphi$ s from mice with LPS treatment was analyzed by immunofluorescence. C, The portion of F4/80 and Rev-erb double-positive cells among the total F4/80 positive cells ( $n=13$ images from 3 mice). Data showed mean \pm SEM of independent experiments. ${ }^{* * *} P<0.001$ 


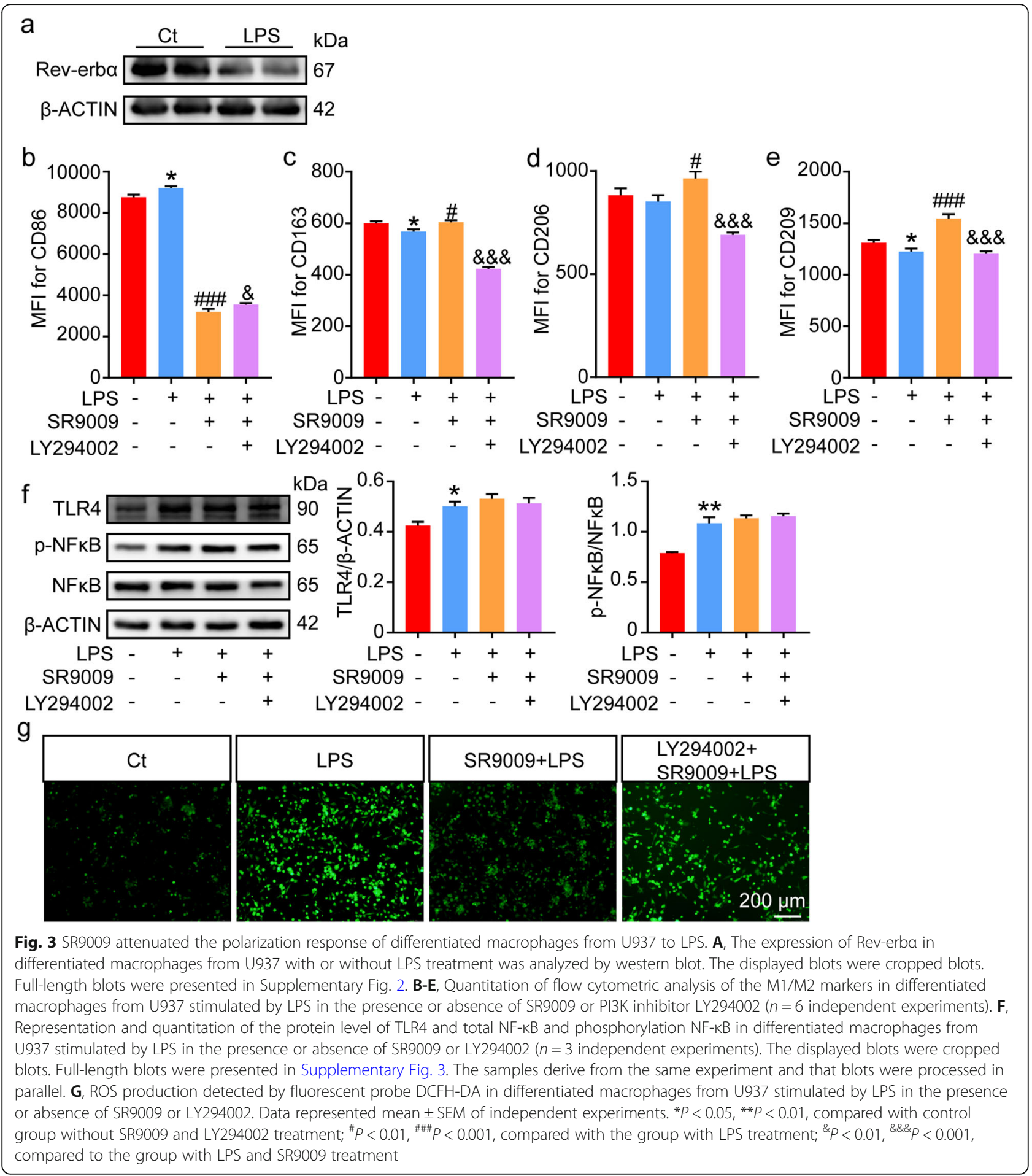

Activation of rev-erba rebalanced M1/M2 polarization of $\mathrm{dM} \varphi s$ and alleviated the abortion rate induced by LPS

The pharmacological activation of Rev-erb $\alpha$ attenuated the effect of LPS on decidual M1/M2 polarization. Whether activation of Rev-erb $\alpha$ prevents LPS-induced pregnant loss needs to be further confirmed. Figure 4AB showed that SR9009 significantly attenuated the embryo resorption rate induced by LPS. Consistent with the results in vitro, the expression of M1 markers (CD11c, CD86 and iNOS) was decreased in $\mathrm{dM} \phi$ s from mice treated with LPS/SR9009 compared to those from mice treated with LPS alone. In contrast, the expression of M2 marker (Arg1) was increased in $\mathrm{dM} \phi$ s from mice treated with LPS/SR9009 compared to those from mice 


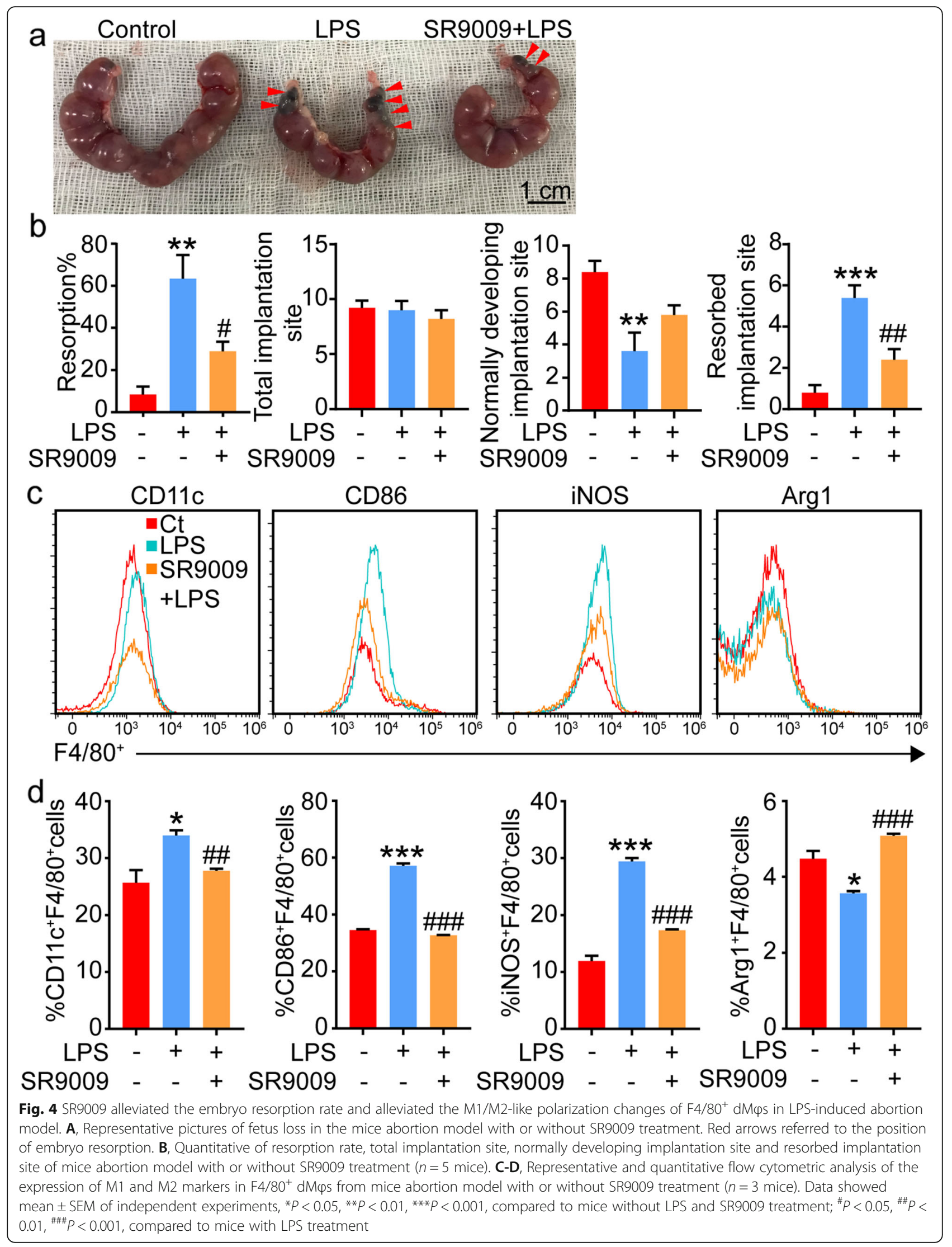


treated with LPS alone (Fig.4C-D). Therefore, the pharmacological activation of Rev-erbo reduced pregnancy loss induced by LPS, accompanied by the rebalance of M1/M2 polarization of $\mathrm{dM} \phi \mathrm{s}$.

\section{Discussion}

Successful pregnancy depends on harmonious microenvironment at the maternal-fetal interface $[28,29]$, where multiple immune cells such as NK cells, macrophages and $\mathrm{T}$ cells with unique phenotypes are present to protect but not attack fetus. Macrophages, as the second abundant immune cells, play important roles in critical biological events of pregnancy establishment and maintenance $[13,30]$. Macrophages display plasticity with different M1/M2 polarization states during distinct pregnancy period. Higher M1/M2 ratio of $\mathrm{dM} \phi \mathrm{s}$ is observed in recurrent spontaneous abortion compared with that in normal pregnancy [31]. In this study, we demonstrated that decreased expression of Rev-erb $\alpha$ and M1 polarization in $\mathrm{dM} \phi$ were displayed in LPS-treated pregnant mice, which was also accompanied by increased embryo resorption rate in early pregnancy. These results suggested that the decreased expression of Rev-erb $\alpha$ might be an important trigger in LPS-induced miscarriage and $\mathrm{M} 1$ polarization.

Miscarriage is one of the common adverse pregnancy outcomes and approximately accounts for one in five pregnancies. It has been proved up to $15 \%$ of early miscarriages and $60 \%$ of late miscarriages were attributed to infection [25]. Many pathogens like bacteria, protozoa and virus can trigger infection, leading to miscarriage $[32,33]$. Bacterial infection can initiate the response of innate immune system. Macrophages play crucial roles in the initiation and resolution of inflammation, which modulate their phenotype in response to environmental signals such as LPS from invading bacteria [34]. LPS is a primary infectious stimulus, which can induce inflammatory response. Recent researches have proved LPS is associated with embryonic abortion and implantation failure in mouse model. Macrophages treated with LPS secrete pro-inflammatory cytokines [19]. During implantation process, trophoblast invasion induces damage of maternal tissue and cell apoptosis, which promoting inflammatory response. However, excessive inflammatory response could induce pregnancy loss such as spontaneous abortion. We also showed that the expression of IFN- $\gamma$ and TNF- $\alpha$ were dramatically increased in decidual tissues from LPS-induced abortion model.

Disruption of circadian rhythm can induce adverse pregnancy outcomes [35]. SCN, as a core pacemaker, transmitted the light entrained rhythm to other nucleus and regulated the secretion of hormones in ovary by hypothalamus-pituitary-ovary axis [36]. Some functions of uterine are regulated by hormone secreted by ovary.
Once the disruption of sleeping, the circadian rhythm in organism is broken and most functions of cells are abnormal [37]. In molecular level, circadian rhythm in cells is modulated by their own transcriptional-translational loop consisted of clock genes. It has been reported that knockout of clock gene such as Bmal1, Clock, Per1 can induce abnormal pregnancy outcomes $[3,10]$. Rev-erb $\alpha$, as an important clock gene in transcriptionaltranslational loop, regulates inflammation, autophagy and metabolism, apart from modulating circadian rhythm. Rev-erb $\alpha$ is reported to inactivate cancer markers, proposing that it could be a potential strategy for cancer treatment [38]. In this study, we demonstrated the expression of Rev-erb $\alpha$ was decreased in both $\mathrm{dM} \phi \mathrm{s}$ and differentiated macrophages from U937 after LPS treatment. Moreover, it has been demonstrated that knockdown of Rev-erb $\alpha$ induced pro-inflammatory response in ESCs [21]. Whether the downregulation of Rev-erb $\alpha$ can strengthen the sensitivity to pathogen stimulation need to be proved in the future.

LPS can trigger the activation of TLR4 and NF-KB signaling pathway. Moreover, upregulation of Rev-erb $\alpha$ can repress the activation of NF- $\mathrm{kB}$ signaling pathway induced by LPS in ESCs [21]. Unexpected, this mechanism was not involved in the regulation of Rev-erba on macrophage polarization. However, PI3K inhibitor can alleviate the role of SR9009 on polarization of differentiated macrophages from U937 with LPS treatment, suggesting PI3k/AKT might be important in the attenuation of LPS-induced decidual M1 polarization. In addition, we suspected the function of other cells which are expressing TLR4 in the implantation site may be disturbed by LPS, and then these cells induced phenotype changes of $\mathrm{dM} \phi \mathrm{s}$, which aggravated the adverse pregnant outcomes. That is to say, the phenotype changes of decidual macrophage may be affected not only directly by LPS but also indirectly by other cells influenced by LPS. In order to confirm the role of decidual macrophages with decreased expression of Rev-erb $\alpha$ on pregnant outcomes, we will construct Rev-erb $\alpha$-conditional-knockout mice to knock out Rev-erb $\alpha$ in macrophages of mice in the future.

The anti-inflammation of Rev-erb $\alpha$ indicated that it could be a potential target for prevention of abortion induced by inflammation. Indeed, the activation of Reverb $\alpha$ decreased LPS-induced embryo resorption rates of pregnant mice. Although SR9009 also alleviated the changes of $\mathrm{M} 1 / \mathrm{M} 2$ polarization of $\mathrm{dM} \phi \mathrm{s}$ from LPStreated mice, this is not the only mechanism of SR9009 in prevention of pregnancy failure. There are many cell types like decidual stromal cells, NK cells and T cells in decidual tissues. LPS can also change the function of these cells, but whether pharmacological activation of Rev-erba reduce the functional changes of these cells 
with LPS treatment need to be further determined in our future study.

In summary, LPS induced inflammatory response and promoted M1-like polarization of macrophages in decidua of mice. Meanwhile, LPS repressed the expression of Reverb $\alpha$ in macrophages. Pharmacological activation of Reverbo using SR9009 may repress M1 polarization of differentiated macrophages from U937 induced by LPS via PI3K signaling pathway. In vivo, SR9009 attenuated LPSinduced abortion rate in mice, and reduced the M1 polarization in macrophages. Our study might supply a potential target for the recurrent spontaneous abortion, especially for inflammation-related miscarriage.

\section{Methods}

\section{Mice and LPS-induced abortion model}

The male and female C57BL/6 mice were purchased from Shanghai SLAC Laboratory Animal Co., Ltd. All mice were bred in room of $22-25^{\circ} \mathrm{C}, 40-60 \%$ relative humidity, $12 \mathrm{~h}$ light- $12 \mathrm{~h}$ dark cycles. All mice experimental procedures were approved by Institutional Animal Care and Use Committee at Fudan University. The female mice were mated with male mice at 19:00. By 7: 00 next morning, vaginal plug was detected and referred as day 0.5 of embryos (E0.5). For abortion model, all the mice were intraperitoneally injected with $0.25 \mathrm{mg} / \mathrm{kg}$ LPS at E7.5. To evaluate the effect of SR9009 on LPSinduced abortion, pregnant female mice were divided into two groups. They were administrated respectively with $50 \mathrm{mg} / \mathrm{kg}$ SR9009 or corn oil by intraperitoneal injection at 16:00 (the time point of peak expression of Rev-erb $\alpha)$ on E6.5 once daily for 5 days. All mice were sacrificed on E13.5 to analyze the abortion rates. All measurements were conducted blind to the group.

\section{Flow cytometry}

In order to obtain single cell suspension, the adherent cells were digested by $0.25 \%$ trypsin with $0.02 \%$ EDTA (Genom, Shanghai, China), and the tissues were digested with Dulbecco's modified Eagle's medium/F-12 (DMEM/ F12) containing $1.0 \mathrm{mg} / \mathrm{ml}$ collagenase IV (Sigma-Aldrich, MO, USA) and $150 \mathrm{U} / \mathrm{ml}$ DNase I (Sigma-Aldrich, MO, USA). The expression of cell-surface and intracellular molecules was detected by flow cytometry. For cellsurface molecules, the cells were incubated with following antibodies: fluorescein isothiocyanate (FITC)-conjugated anti-mouse F4/80; PerCP/Cyanine5.5 anti-mouse CD11c; Brilliant Violet (BV) 421-conjugated anti-mouse CD86; (phycoerythrin (PE)-conjugated anti-human CD163; allophycocyanin (APC)-conjugated anti-human CD206; PE/ Cyanine7 anti-human CD86; PerCP/Cyanine5.5 antihuman CD209 (Biolegend, CA, USA). For intracellular molecules tests, the cells were fixed and permeabilized with Fix/Perm Kit (Biolegend, CA, USA) and then incubated with antibodies: APC-eFluor 780-conjugated anti-mouse iNOS (Ebioscience, CA, USA); PE-conjugated anti-mouse Arg1 (Invitrogen, MA, USA)). A minimum of 10,000 events were collected by a BD or Beckman flow cytometer and analyzed with FlowJo or CytExpert software.

\section{Quantitative real-time polymerase chain reaction (qPCR)}

The RNA in adherent cells or tissues was extracted by TRIzol reagent (Takara, Honshu, Japan) according to manufacturer's instruction. $1 \mu \mathrm{g}$ RNA was reversetranscribed into complementary DNA (cDNA), which was amplified with SYRB Green PCR Master Mix (Takara, Honshu, Japan) on ABI PRISM 7900 Sequence Detection System (Applied Biosystems, MA, USA). $\beta$-actin (Actb) was used as an internal control to normalize relative changes in gene expression through $2^{-\Delta \Delta \mathrm{Ct}}$ method. The specific primers were as follows: IL- $1 \beta$, forward $5^{\prime}$ - AATG CCACCTTTTGACAGTGATG-3' and reverse 5' - AGCT TCTCCACAGCCACAAT-3'; IL-6, forward 5' - ATCCAGTTGCCTTCTTGGGACTGA-3' and reverse 5'TAAGCCTCCGACTTGTGAAGTGGT-3'; TNF- $\alpha$, forward 5' - AGGGTCTGGGCCATAGAACT-3' and reverse 5' - CCACCACGCTCTTCTGTCTAC-3'; IFN- $\gamma$, forward 5' - GCTACACACTGCATCTTGGC - 3' and reverse 5'CATGTCACCATCCTTTTGCCAG-3'; IL-17a, forward 5' - TTTAACTCCCTTGGCGCAAAA - $3^{\prime}$ and reverse 5' - CTTTCCCTCCGCATTGACAC -3'; IL-5, forward $5^{\prime}$ - CTCTGTTGACAAGCAATGAGACG $-3^{\prime}$ and reverse $5^{\prime}$ - TCTTCAGTATGTCTAGCCCCTG - 3'; TGF $\beta$, forward $5^{\prime}$ - CTCCCGTGGCTTCTAGTGC -3 ' and reverse $5^{\prime}$ - GCCTTAGTTTGGACAGGATCTG - 3'.

\section{Western blot assay}

The whole proteins were extracted from homogenized cells and tissues using radioimmunoprecipitation assay lysis buffer (Beyotime, Shanghai, China) containing phosphatase repressor (Roche, Basel, Switzerland) and protease inhibitor (Beyotime, Shanghai, China) based on previous publication [21]. Samples containing $20 \mu \mathrm{g}$ proteins were separated by $10 \%$ sodium dodecyl sulfonate polyacrylamide gel. Proteins were transferred onto polyvinylidene difluoride membrane (Millipore, Darmstadt, Germany) and then incubated with primary antibodies (anti-Rev-erba (Santa Cruze, TX, USA); anti-TLR4 (Abcam, CA, USA); anti-p-NF- $\kappa B$ (Cell Signaling Technology, MA, USA); anti-NF- $\mathrm{kB}$ (Cell Signaling Technology, MA, USA); anti- $\beta$-ACTIN (Abcam, CA, USA)) after blocked with $5 \%$ non-fat dry milk powder in trisbuffered saline (TBS) with $0.1 \%$ Tween 20 (TBST). The membrane was washed with TBST for 4 times $(5 \mathrm{~min}$ at every turn) and incubated with secondary antibodies. The membrane was visualized by an enhanced chemiluminescence detection system. $\beta$-ACTIN was used to 
normalize the protein expression by greyscale analysis using ImageJ software.

\section{Immunofluorescence}

Mouse decidual tissues were fixed using paraformaldehyde and then prepared for paraffin section. Paraffin sections was applied with citrate sodium solution for antigen retrieval after dewaxed using dimethylbenzene and ethanol with different concentrations. The slices were sealed with $0.05 \%$ TritonX-100 and 10\% donkey serum and then incubated with primary antibodies (anti-F4/80 (Invitrogen, MA, USA); anti-Rev-erb $\alpha$ (Santa Cruze, TX, USA)) for overnight at $4{ }^{\circ} \mathrm{C}$. After washed by TBS for three times, the slices were incubated with secondary antibodies for 2 $\mathrm{h}$ at room temperature. Next, the slices were stained with 4',6-diamidino-2-phenylindole (DAPI) for $7 \mathrm{~min}$ and then washed with TBS for 3 times. At last, the slices were sealed with mounting medium and photographed using a fluorescence microscope.

\section{Cell culture and treatment}

U937 cells, human monocyte cell line, were cultured with complete medium (RPMI1640 supplemented with $10 \%$ fetal bovine serum (FBS), $100 \mathrm{U} / \mathrm{mL}$ penicillin and $100 \mu \mathrm{g} / \mathrm{mL}$ streptomycin (Sangon Biotech, Shanghai, China) in a $37^{\circ} \mathrm{C}$ humidified incubator containing 5\% $\mathrm{CO}_{2}$. To obtain differentiated macrophages from U937, U937 cells were dealt with $100 \mathrm{ng} / \mathrm{ml}$ phorbol 12myristate 13-acetate (PMA) (Sigma-Aldrich, MO, USA) for $24 \mathrm{~h}$. The polarization status of differentiated macrophages from U937 were analyzed after $100 \mathrm{ng} / \mathrm{ml}$ LPS (Sigma-Aldrich, MO, USA) treatment for $48 \mathrm{~h}$. For analysis of Rev-erb $\alpha$ activation, differentiated macrophages from U937were dealt with $10 \mu \mathrm{M}$ SR9009 for $4 \mathrm{~h}$ before LPS treatment. For signal pathway analysis, differentiated macrophages from U937 were treated with LY294002 (MedChemExpress, NJ, USA) for $1 \mathrm{~h}$ before SR9009 treatment.

\section{Reactive oxygen species}

The treated cells dealt with serum-free RPMI1640 medium supplemented with $1 \mu \mathrm{M} \quad 2^{\prime}, 7^{\prime}$-dichlorohydrofluorescin diacetate (DCFH-DA) (Sigma-Aldrich, MO, USA) for $30 \mathrm{~min}$ at $37^{\circ} \mathrm{C}$. And then the cells were washed three times with serum-free RPMI1640 medium. The cells were taken pictures using a fluorescence microscope.

\section{Statistical analysis}

All statistics were assessed by GraphPad Prism Version 7 and were presented as mean \pm standard error of the mean (SEM). Comparison between the two groups was analyzed by Student's t-test. Multiple groups were analyzed by ANOVA. $P<0.05$ was defined as statistically significant difference.

\section{Abbreviations}

LPS: lipopolysaccharide; SCN: suprachiasmatic nuclei; Bmal1: Brain and muscle ARNT-like 1; Clock: circadian locomotor output cycles kaput; $\mathrm{dM} \varphi$ s: decidual macrophages; NK: natural killer; IFN- $\gamma$ : interferon- $\gamma ;$ TNFa: tumor necrosis factor-a; IL: interleukin; TLR: Toll-like receptor; ESCs: endometrial stroma cells; SEM: standard error of the mean; qPCR: quantitative real-time polymerase chain reaction; TBS: tris-buffered saline; DAPI: 4',6-diamidino-2-phenylindole; FBS: fetal bovine serum; DCFHDA: 2',7'-dichlorohydrofluorescin diacetate

\section{Supplementary Information}

The online version contains supplementary material available at https://doi. org/10.1186/s12865-021-00438-4

Additional file 1: Fig. S1. Uncropped full-length blots with high contrast and low contrast were included for Fig. 2A.

Additional file 2: Fig. S2. Uncropped full-length blots with high contrast and low contrast were included for Fig. 3A.

Additional file 3: Fig. S3. Uncropped full-length blots with high contrast and low contrast were included for Fig. 3F. The samples derive from the same experiment with and that blots were processed in parallel. *The blot was not shown in Fig. 3F.

\section{Acknowledgments}

Not applicable.

\section{Authors' contributions}

L.C. designed this project, performed experiments, analyzed data and drafted the manuscript. F.X. performed experiments. S.W. helped to analyze data. X.L. and H.L. contributed to performed experiments. Y.D. took part in discussing this project. M.D. conceived this project and revised this manuscript. All authors reviewed and approved the final manuscript.

\section{Funding}

This work was supported by the National Key R\&D Program of China (2017YFC1001403), National Natural Science Foundation of China (31970859, 81630036, 91542116, 81871170), The Strategic Collaborative Research Program of the Ferring Institute of Reproductive Medicine Supported by Ferring Pharmaceuticals and Chinese Academy of Sciences (FIRMX200504), Shanghai Sailing Program (19YF1404100), the Program of Shanghai Academic/Technology Research Leader (17XD1400900), the Innovationoriented Science and Technology Grant from NHC Key Laboratory of Reproduction Regulation (CX2017-2) and Innovative research team of highlevel local universities in Shanghai.

Availability of data and materials

The datasets analyzed in the current study are available from the corresponding author on reasonable request.

\section{Declarations}

\section{Ethics approval and consent to participate}

The study is reported in accordance with ARRIVE guidelines. All animal protocols were approved by Institutional Animal Care and Use Committee at Fudan University, the ethics committee approval code was FE20053. This study does not involve the use of human data or tissue. All experiments were performed in accordance with relevant guidelines and regulations at Obstetrics and Gynecology Hospital of Fudan University. All mice were sacrificed by exsanguination under anesthesia by $5 \%$ isoflurane. The sacrificed mice were handed over to special departments for unified treatment. All procedures were approved by Institutional Animal Care and Use Committee at Fudan University.

Consent for publication

Not applicable.

Competing interests

All authors have not any conflicts of interest to declare. 


\section{Author details}

${ }^{1} \mathrm{NHC}$ Key Lab of Reproduction Regulation (Shanghai Institute of Planned Parenthood Research), Hospital of Obstetrics and Gynecology, Fudan University Shanghai Medical College, Shanghai 200090, China. ${ }^{2}$ Shanghai Key Laboratory of Female Reproductive Endocrine Related Diseases, Shanghai 200090, China. ${ }^{3}$ Key Laboratory of Zoological Systematics and Evolution, Institute of Zoology, Chinese Academy of Sciences, Beijing 100101, China. ${ }^{4}$ Hospital of Obstetrics and Gynecology, Fudan University Shanghai Medical College, Fangxie Road 419, Shanghai 200011, China. ${ }^{5}$ Department of Obstetrics and Gynecology, Guangzhou First People's Hospital, School of Medicine, South China University of Technology, Guangzhou 510180, China. 'Laboratory for Reproductive Immunology, Hospital of Obstetrics and Gynecology, Fudan University Shanghai Medical College, ZhaoZhou Road 413, Shanghai 200011, China.

Received: 29 December 2020 Accepted: 13 July 2021

Published online: 16 August 2021

\section{References}

1. Metz RP, Qu X, Laffin B, Earnest D, Porter WW. Circadian clock and cell cycle gene expression in mouse mammary epithelial cells and in the developing mouse mammary gland. Dev Dyn. 2006;235(1):263-71. https://doi.org/10.1 002/dvdy.20605

2. Herzog ED, Hermanstyne T, Smyllie NJ, Hastings MH: Regulating the Suprachiasmatic Nucleus (SCN) Circadian Clockwork: Interplay between CellAutonomous and Circuit-Level Mechanisms. Cold Spring Harb Perspect Biol. 2017:9(1):a027706.

3. Liu Y, Johnson BP, Shen AL, Wallisser JA, Krentz KJ, Moran SM, et al. Loss of BMAL1 in ovarian steroidogenic cells results in implantation failure in female mice. Proc Natl Acad Sci U S A. 2014;111(39):14295-300. https://doi. org/10.1073/pnas.1209249111.

4. Ramakrishnan SN, Muscat GE. The orphan rev-erb nuclear receptors: a link between metabolism, circadian rhythm and inflammation? Nucl Recept Signal. 2006;: $: 009$.

5. Bugge A, Feng D, Everett LJ, Briggs ER, Mullican SE, Wang F, et al. Reverbalpha and rev-erbbeta coordinately protect the circadian clock and normal metabolic function. Genes Dev. 2012;26(7):657-67. https://doi.org/1 $0.1101 / \mathrm{gad} .186858 .112$.

6. Huang $\mathrm{G}$, Zhang $\mathrm{F}, \mathrm{Ye} \mathrm{Q}$, Wang $\mathrm{H}$. The circadian clock regulates autophagy directly through the nuclear hormone receptor $\mathrm{Nr} 1 \mathrm{~d} 1 /$ rev-erbalpha and indirectly via Cebpb/(C/ebpbeta) in zebrafish. Autophagy. 2016;12(8):1292309. https://doi.org/10.1080/15548627.2016.1183843.

7. Wang S, Lin Y, Yuan X, Li F, Guo L, Wu B. REV-ERBalpha integrates colon clock with experimental colitis through regulation of NF-kappaB/NLRP3 axis. Nat Commun. 2018;9(1):4246. https://doi.org/10.1038/s41467-018-06568-5.

8. Zhu JL, Hjollund NH, Olsen J. National Birth Cohort in D: shift work, duration of pregnancy, and birth weight: the National Birth Cohort in Denmark. Am J Obstet Gynecol. 2004;191(1):285-91. https://doi.org/10.1 016/j.ajog.2003.12.002.

9. Kloss JD, Perlis ML, Zamzow JA, Culnan EJ, Gracia CR. Sleep, sleep disturbance, and fertility in women. Sleep Med Rev. 2015;22:78-87. https:// doi.org/10.1016/.smrv.2014.10.005.

10. Li R, Cheng S, Wang Z. Circadian clock gene plays a key role on ovarian cycle and spontaneous abortion. Cell Physiol Biochem. 2015;37(3):911-20. https://doi.org/10.1159/000430218.

11. Nakamura TJ, Moriya T, Inoue S, Shimazoe T, Watanabe S, Ebihara S, et al. Estrogen differentially regulates expression of Per1 and Per2 genes between central and peripheral clocks and between reproductive and nonreproductive tissues in female rats. J Neurosci Res. 2005;82(5):622-30. https://doi.org/10.1002/jnr.20677.

12. Kimura F, Takebayashi A, Ishida M, Nakamura A, Kitazawa J, Morimune A et al. Review: chronic endometritis and its effect on reproduction. J Obstet Gynaecol Res. 2019;45(5):951-60. https://doi.org/10.1111/jog.13937.

13. Ning F, Liu H, Lash GE. The role of Decidual macrophages during Normal and pathological pregnancy. Am J Reprod Immunol. 2016;75(3):298-309. https://doi.org/10.1111/aji.12477.

14. Li Y, Xie Z, Wang Y, Hu H. Macrophage M1/M2 polarization in patients with pregnancy-induced hypertension. Can J Physiol Pharmacol. 2018;96(9):922-8. https://doi.org/10.1139/cjpp-2017-0694.

15. Zhang $\mathrm{YH}$, He M, Wang $\mathrm{Y}$, Liao AH. Modulators of the balance between $\mathrm{M} 1$ and M2 macrophages during pregnancy. Front Immunol. 2017;8:120.
16. Keller M, Mazuch J, Abraham U, Eom GD, Herzog ED, Volk HD, et al. A circadian clock in macrophages controls inflammatory immune responses. Proc Natl Acad Sci U S A. 2009;106(50):21407-12. https://doi.org/10.1073/ pnas.0906361106

17. Lam MT, Cho H, Lesch HP, Gosselin D, Heinz S, Tanaka-Oishi Y, et al. RevErbs repress macrophage gene expression by inhibiting enhancer-directed transcription. Nature. 2013;498(7455):511-5. https://doi.org/10.1038/na ture12209.

18. Sato S, Sakurai T, Ogasawara J, Takahashi M, Izawa T, Imaizumi K, et al. A circadian clock gene, rev-erbalpha, modulates the inflammatory function of macrophages through the negative regulation of $\mathrm{C} / 2$ expression. J Immunol. 2014;192(1):407-17. https://doi.org/10.4049/jimmunol.1301982.

19. Wang $Y$, Mao X, Chen $\mathrm{H}$, Feng J, Yan M, Wang $Y$, et al. Dexmedetomidine alleviates LPS-induced apoptosis and inflammation in macrophages by eliminating damaged mitochondria via PINK1 mediated mitophagy. Int Immunopharmacol. 2019;73:471-81. https://doi. org/10.1016/j.intimp.2019.05.027.

20. Zhou M, Yi Y, Hong L. Oridonin ameliorates lipopolysaccharide-induced endometritis in mice via inhibition of the TLR-4/NF-kappaBpathway. Inflammation. 2019;42(1):81-90. https://doi.org/10.1007/s10753-018-0874-8.

21. Zhao W, Cui L, Huang X, Wang S, Li D, Li L, et al. Activation of rev-erbalpha attenuates lipopolysaccharide-induced inflammatory reactions in human endometrial stroma cells via suppressing TLR4-regulated NF-kappaB activation. Acta Biochim Biophys Sin Shanghai. 2019;51(9):908-14. https:// doi.org/10.1093/abbs/gmz078.

22. Huang J, Xue M, Zhang J, Yu H, Gu Y, Du M, et al. Protective role of GPR120 in the maintenance of pregnancy by promoting decidualization via regulation of glucose metabolism. EBioMedicine. 2019;39:540-51. https:// doi.org/10.1016/j.ebiom.2018.12.019.

23. Wu H, Zhao G, Jiang K, Li C, Qiu C, Deng G. Engeletin alleviates lipopolysaccharide-induced endometritis in mice by inhibiting TLR4mediated NF-kappaB activation. J Agric Food Chem. 2016;64(31):6171-8. https://doi.org/10.1021/acs.jafc.6b02304.

24. Liu L, Guo H, Song A, Huang J, Zhang Y, Jin S, et al. Progranulin inhibits LPS-induced macrophage M1 polarization via NF-small ka, CyrillicB and MAPK pathways. BMC Immunol. 2020;21(1):32. https://doi.org/10.1186/s12 865-020-00355-y.

25. Giakoumelou S, Wheelhouse N, Cuschieri K, Entrican G, Howie SE, Horne AW. The role of infection in miscarriage. Hum Reprod Update. 2016;22(1): 116-33. https://doi.org/10.1093/humupd/dmv041.

26. Lu J, Xie L, Liu C, Zhang Q, Sun S. PTEN/PI3K/AKT regulates macrophage polarization in emphysematous mice. Scand J Immunol. 2017;85(6):395-405. https://doi.org/10.1111/sji.12545.

27. Vergadi $E$, leronymaki $E$, Lyroni $K$, Vaporidi $K$, Tsatsanis C. Akt signaling pathway in macrophage activation and M1/M2 polarization. J Immunol. 2017;198(3):1006-14. https://doi.org/10.4049/jimmunol.1601515.

28. Bonney EA. Alternative theories: pregnancy and immune tolerance. J Reprod Immunol. 2017;123:65-71. https://doi.org/10.1016/j.jri.2017.09.005.

29. Okada H, Tsuzuki T, Murata H. Decidualization of the human endometrium. Reprod Med Biol. 2018;17(3):220-7. https://doi.org/10.1002/rmb2.12088.

30. Faas MM, de Vos P. Uterine NK cells and macrophages in pregnancy. Placenta. 2017;56:44-52. https://doi.org/10.1016/j.placenta.2017.03.001.

31. Sheng YR, Hu WT, Wei CY, Tang LL, Liu YK, Liu YY, et al. IL-33/ST2 axis affects the polarization and efferocytosis of decidual macrophages in early pregnancy. Am J Reprod Immunol. 2018;79(6):e12836. https://doi.org/1 0.1111/aji.12836

32. Hudalla H, Karenberg K, Kuon RJ, Poschl J, Tschada R, Frommhold D. LPSinduced maternal inflammation promotes fetal leukocyte recruitment and prenatal organ infiltration in mice. Pediatr Res. 2018;84(5):757-64. https:// doi.org/10.1038/s41390-018-0030-z.

33. Christoffersen M, Troedsson M. Inflammation and fertility in the mare. Reprod Domest Anim. 2017;52(Suppl 3):14-20. https://doi.org/10.1111/ rda.13013.

34. Duriez M, Quillay H, Madec Y, El Costa H, Cannou C, Marlin R, et al. Human decidual macrophages and NK cells differentially express toll-like receptors and display distinct cytokine profiles upon TLR stimulation. Front Microbiol. 2014;5:316.

35. Boden MJ, Kennaway DJ. Circadian rhythms and reproduction. Reproduction. 2006;132(3):379-92. https://doi.org/10.1530/rep.1.00614.

36. Smarr BL, Morris E, de la Iglesia HO. The dorsomedial suprachiasmatic nucleus times circadian expression of Kiss 1 and the luteinizing hormone 
surge. Endocrinology. 2012;153(6):2839-50. https://doi.org/10.1210/en.2 011-1857.

37. Knutsson A. Health disorders of shift workers. Occup Med (Lond). 2003;53(2): 103-8. https://doi.org/10.1093/occmed/kgg048.

38. Sulli G, Rommel A, Wang X, Kolar MJ, Puca F, Saghatelian A, et al. Pharmacological activation of REV-ERBs is lethal in cancer and oncogeneinduced senescence. Nature. 2018;553(7688):351-5. https://doi.org/10.1038/ nature25170.

\section{Publisher's Note}

Springer Nature remains neutral with regard to jurisdictional claims in published maps and institutional affiliations.

Ready to submit your research? Choose BMC and benefit from:

- fast, convenient online submission

- thorough peer review by experienced researchers in your field

- rapid publication on acceptance

- support for research data, including large and complex data types

- gold Open Access which fosters wider collaboration and increased citations

- maximum visibility for your research: over $100 \mathrm{M}$ website views per year

At $\mathrm{BMC}$, research is always in progress.

Learn more biomedcentral.com/submissions 